\title{
EFECTOS DE UN PROGRAMA DE ACTIVIDAD FÍSICA EN LA COMPOSICIÓN CORPORAL DE ESCOLARES DE 3-5 AÑOS
}

\section{EFFECTS OF A PHYSICAL ACTIVITY PROGRAM ON BODY COMPOSITION OF SCHOOL CHILDREN OF 3-5 YEARS}

\author{
López Sánchez, G.F. ${ }^{1}$, Borrego Balsalobre, F.J. ${ }^{1}$, Dí az Suárez, A. ${ }^{1}$ \\ guillels23@hotmail.com \\ ${ }^{1}$ Facultad de Ciencias del Deporte, Universidad de Murcia, España. \\ Recibido: Junio/2013 - Aceptado: Noviembre/2013.
}

\begin{abstract}
Resumen
Objetivos: Este artículo se centra en estudiar los efectos de un programa de actividad física sobre la composición corporal de un grupo de escolares. Método: Han participado 36 escolares (20 niños y 16 niñas), entre los 3 y los 5 años de edad. Las variables estudiadas han sido: masa grasa, masa libre de grasa, IMC (Índice de Masa Corporal), ICC (Indice Cintura/Cadera). El análisis de la composición corporal se ha realizado mediante bioimpedancia eléctrica, por medio del Monitor de grasa corporal Tanita BC 418-MA Segmental. El procedimiento ha sido: pre-test, intervención y pos-test. La intervención ha consistido en 3 días a la semana de actividad física de alta intensidad, 15 minutos al día, durante 12 semanas. Resultados y conclusiones: Mejoras significativas en la masa grasa (Sig. $<0.05$ ). No se han encontrado mejoras significativas en la masa libre de grasa, el IMC y el ICC.
\end{abstract}

Palabras clave: Composición Corporal, Actividad Física, Educación Física, Educación Infantil.

\begin{abstract}
Objectives: This paper focuses on studying the effects of a physical activity program on body composition of a group of schoolchildren. Method: This investigation involved 36 students ( 20 boys and 16 girls), aged between 3 and 5 years. The variables considered were: fat mass, fat-free mass, BMI (Body Mass Index), WHR (Waist to Hip Ratio). The body composition analysis was performed using bioelectrical impedance through the body fat monitor Tanita BC 418-MA Segmental. The procedure was as follows: pre-test, intervention and post-test. The intervention consisted of 3 days per week of high-intensity physical activity, 15 minutes per day, during 12 weeks. Results and Conclusions: Significant improvements in fat mass (Sig $<0.05$ ). No significant improvements in fat-free mass, BMI and WHR.
\end{abstract}

Keywords: Body Composition, Physical Activity, Physical Education, Childhood Education.

\section{INTRODUCCIÓN}

La composición corporal (CC) es un concepto ampliamente estudiado, y que ha demostrado ser uno de los parámetros que más estrechamente se relacionan con el estado nutricional, así como, con la salud de las personas (Alvero et al., 2010).

La composición corporal se puede definir como el fraccionamiento del peso corporal en compartimentos (Berral et al., 1991). Por tanto, el análisis de la composición corporal permite conocer las proporciones de los distintos constituyentes del cuerpo humano (Moreno, 2000).

Según Moreno (2000), el modelo tradicionalmente usado para evaluar la composición corporal es el modelo de dos-compartimentos o bicompartimental, que considera que el cuerpo humano está compuesto por una parte de masa grasa y otra parte de masa libre de grasa; o de modo alternativo, masa grasa y masa magra. No obstante, Aguado \& Gómez (2005) señalan que en función del modelo de análisis de la composición corporal deben ser usadas diferentes técnicas.

La medición de la CC permite obtener una información importante sobre el estado de salud de la población estudiada, así como detectar precozmente algunas enfermedades (Alburquerque, 2008). Algunos estudios han encontrado relaciones entre la CC y el envejecimiento, la obesidad, la diabetes mellitus tipo 2 (Oria et al, 2002) y patologías tales como el cáncer o el SIDA (Laskey, 1996). Además, existe una estrecha relación entre el exceso de grasa corporal y el riesgo de aparición de enfermedades cardiovasculares (Björntorp, 1992; Roche, 1992).

La composición corporal también se relaciona con otros parámetros saludables como actividad física habitual (Plonka et al., 2011), calidad de la dieta (Donnelly et al., 1991), calidad de vida (Lebrun et al., 2006), imagen corporal (Streeter et al., 2012), glucosa, colesterol, triglicéridos, GPT, tensión arterial (Rosenfalck et al., 1996; Wu \& Tai, 1990) y frecuencia cardiaca (Bassey et al., 1979).

Debido a la relación directa de la composición corporal con el estado de salud de las personas, son necesarias intervenciones encaminadas a mejorar la composición corporal de las personas y, en consecuencia, su salud.

El principal objetivo de esta investigación es determinar los efectos producidos en la composición corporal de escolares de 3 a 5 años tras una intervención mediante actividad física intensa de 12 semanas de duración. La hipótesis de partida es que, tras la intervención propuesta, se producirán mejoras significativas en la composición corporal de los escolares.

\section{MÉTODO}

\section{Muestra}

Un total de 36 escolares de 3 a 5 años han participado en el estudio. Los escolares pertenecen a la etapa educativa 
de Educación Infantil. En función del sexo, hay 20 niños y 16 niñas. En función del curso, hay 9 (6 niños y 3 nińas) de primer curso (3 ańos), 6 ( 4 nińos y 2 nińas) de segundo curso (4 años) y 21 (10 niños y 11 niñas) de tercer curso (5 años). Esta investigación ha sido aprobada por la Comisión de Ética de Investigación de la Universidad de Murcia y todos los padres o tutores de los participantes han firmado un consentimiento informado.

\section{Diseño}

El diseño ha sido un diseño cuasiexperimental prepost con un solo grupo de intervención, ya que los alumnos participantes fueron seleccionados por el interés de sus padres en el programa de actividad física planteado, de forma no aleatoria, y se consideró como aspecto primordial de la investigación que todos los alumnos pudieran participar en el programa de actividad física.

Los sujetos participantes en el estudio han realizado un pre-test, una intervención de 12 semanas y un postest.

En el pre-test se ha analizado la composición corporal de los escolares: masa grasa, masa libre de grasa, IMC (Índice de Masa Corporal), ICC (Î́ndice Cintura/Cadera).

Durante la intervención, los escolares participantes han realizado tres veces a la semana sesiones de actividad física de 15 minutos de duración de intensidad alta. Las sesiones han sido dirigidas por el personal investigador. El lugar donde se ha llevado a cabo la intervención ha sido el patio y la pista del centro escolar, en horario escolar. La intervención se ha realizado dividiendo a los alumnos según su edad (un grupo de 3 años, un grupo de 4 años y otro de 5 años), en momentos diferentes y realizando adaptaciones a las actividades de la intervención según la edad. Las actividades utilizadas en la intervención han tenido como prioridad que los alumnos realicen actividad física intensa durante 15 minutos seguidos, con actividades como carreras, persecuciones, laberintos, relevos con grupos muy reducidos...

En el pos-test se ha vuelto a analizar la composición corporal de los escolares para comprobar si se han producido mejoras respecto al pre-test.

\section{Análisis de la Composición Corporal}

Se ha analizado mediante Impedancia Bioeléctrica con el Analizador de la Composición Corporal Tanita BC 418-MA Segmental. Se han seguido todas las recomendaciones para realizar el análisis de la Impedancia Bioeléctrica. Este analizador de la composición corporal permite conocer el Peso, la Masa Grasa y la Masa Libre de Grasa (total y por segmentos). Además se han calculado el Î́ndice de Masa Corporal y el Índice Cintura/Cadera. Por tanto, también se han utilizado un tallímetro para medir la talla y una cinta métrica para medir los perímetros de cintura y cadera.

\section{Análisis Estadístico}

Se ha realizado un análisis estadístico por medio del Statistical Package for Social Sciences 15.0 (SPSS-15.0). Se han seguido las indicaciones del Manual de estadística aplicada a las Ciencias de la Actividad Física y el Deporte (Ortega, Ortiz \& Artés, 2009). Se ha llevado a cabo el siguiente análisis estadístico:

- Prueba de normalidad (Kolmogorov-Smirnov) para verificar la hipótesis de que la muestra procede de una distribución normal.

- Prueba T para muestras relacionadas para saber si se han producido diferencias significativas entre el pre-test y el pos-test.

\section{RESULTADOS}

A continuación se presentan los resultados en dos tablas:

En la Tabla 1 se presenta una clasificación de la muestra en función del IMC, clasificando a la muestra en peso insuficiente, normopeso, sobrepeso y obesidad. En esta tabla se pueden observar los datos según el sexo $\mathrm{y}$ los datos totales.

En la Tabla 2 se resumen los resultados de la investigación, comparando las medias de las diferentes variables en el pretest y el postest e indicando el grado de significatividad. Cuando hay diferencias significativas entre el pretest y el postest $(\mathrm{p}<0.05)$ se indica con un doble asterisco $\left({ }^{* *}\right)$.

Tabla 1. Clasificación de la muestra según IMC, N=36

\begin{tabular}{|l|c|c|c|c|c|c|}
\hline \multirow{2}{*}{} & \multicolumn{2}{|c|}{ Niños } & \multicolumn{2}{c|}{ Niñas } & \multicolumn{2}{c|}{ Total } \\
\cline { 2 - 7 } & $n$ & $\%$ & $n$ & $\%$ & $n$ & $\%$ \\
\hline Peso insuficiente & 1 & $5 \%$ & 0 & $0 \%$ & 1 & $2.8 \%$ \\
\hline Normopeso & 14 & $70 \%$ & 6 & $37.5 \%$ & 20 & $55.6 \%$ \\
\hline Sobrepeso & 5 & $25 \%$ & 2 & $12.5 \%$ & 7 & $19.4 \%$ \\
\hline Obesidad & 0 & $0 \%$ & 8 & $50 \%$ & 8 & $22.2 \%$ \\
\hline
\end{tabular}




\begin{tabular}{|l|l|l|l|l|}
\hline Variable & $\begin{array}{l}\text { Media } \\
\text { pretest }\end{array}$ & $\begin{array}{l}\text { Media } \\
\text { postest }\end{array}$ & Dif. medias & Significativ. \\
\hline Masa Grasa (\%) & 22,525 & 20,844 & 1,6806 & $0,000 \quad$ (*) \\
\hline Masa Libre de Grasa (\%) & 77,486 & 77,847 & $-0,3611$ & 0,794 \\
\hline IMC & 16,972 & 17,194 & $-0,222$ & 0,088 \\
\hline ICC & 0,8844 & 0,8861 &,- 0017 & 0,874 \\
\hline
\end{tabular}

\section{DISCUSIÓN}

Respecto a la Tabla 1 y tomando como referencia los valores de la OMS (2013), se observa que el 19.4\% de los escolares de la muestra tienen sobrepeso y el $22.4 \%$ obesidad. Estos datos contrastan con otros estudios como el de Espín Ríos et al. (2013), llevado a cabo en una muestra de 43143 niños de 3 a 5 años de la Región de Murcia y que arroja los siguientes resultados: 3 años (15\% sobrepeso y 7\% obesidad), 4 años $(16,6 \%$ sobrepeso y $8.5 \%$ obesidad) y 5 años (17.6\% sobrepeso y $13.4 \%$ obesidad). En relación a los datos nacionales, el INE (2012) con una muestra de 1.510.000 niños de 2 a 4 años dio las siguientes cifras para esta franja de edad: $11,26 \%$ sobrepeso y $18,81 \%$ obesidad. Además estos datos pueden ser comparados con estudios realizados en otros países como el de Velásquez et al. (2008), que con una muestra de 238 niños chilenos de 3 a 5 años de edad, encontró un $22 \%$ de obesos y un $38 \%$ de sobrepeso. Por último, es interesante la comparación con el estudio de Logan et al. (2011), que con una muestra de 38 niños de 4 a 6 años de Delaware, observó un $7.9 \%$ de obesidad y un $13.2 \%$ de sobrepeso.

Continuando con la Tabla 1 y en función del sexo cabe destacar que el $62.5 \%$ de las niñas de la muestra presentan obesidad o sobrepeso, frente a solo un $25 \%$ de los niños. Estos datos están en la línea de los datos regionales aportados por Espín Ríos et al. (2013), que también informan de mayores porcentajes de sobrepeso y obesidad en las niñas de esta edad: 3 años (niños $13.5 \%$ sobrepeso y $6.3 \%$ obesidad; niñas $16.6 \%$ sobrepeso y $7.8 \%$ obesidad), 4 años (niños: $14.5 \%$ sobrepeso y $7.7 \%$ obesidad; niñas: $18.7 \%$ sobrepeso y $9.4 \%$ obesidad) y 5 años (niños: $16.2 \%$ sobrepeso y $12.6 \%$

\section{REFERENCIAS}

Aguado Henche, S. \& Gómez Pellico, L. (2005). Body composition: evaluation methods. European Journal of Anatomy, 9(2), 117-124.

Alburquerque Sendín, F. (2008). Estudio comparativo intermetodológico de la composición obesidad; nińas: $19.1 \%$ sobrepeso y $14.2 \%$ obesidad). No obstante, nuestros datos difieren con los nacionales (INE, 2012), que indica mayores niveles de sobrepeso y obesidad en los niños de esta edad: $12.12 \%$ sobrepeso y $18.97 \%$ obesidad en niños frente a $10.27 \%$ sobrepeso y $18.64 \%$ en niñas.

En referencia ahora a la Tabla 2 y en relación a los cambios entre el pretest y el postest, los resultados obtenidos indican que se han producido mejoras significativas en el porcentaje de masa grasa $(\mathrm{p}<0.05)$. No obstante no se han producido mejoras significativas en el porcentaje de masa libre de grasa $(\mathrm{p}=0,794)$, el $\operatorname{IMC}(\mathrm{P}=0,088)$ y el ICC $(\mathrm{P}=0,874)$. No se han encontrado en la literatura intervenciones mediante actividad física similares en esta franja de edad con las que poder comparar nuestros resultados.

\section{CONCLUSIONES}

Tras la intervención se han conseguido mejoras significativas en la masa grasa de los escolares pero no en la masa libre de grasa, el IMC y el ICC.

Las principales limitaciones de la investigación han sido la escasez de la muestra y el reducido tiempo diario de intervención.

En futuras investigaciones se recomienda llevar a cabo otros programas de intervención mediante actividad física, con muestras más amplias y mayor duración. Asimismo, se aconseja utilizar diferentes tipos e intensidades de actividad física y ampliar el rango de edad de la muestra. De esta forma habrá más recursos disponibles para mejorar la composición corporal de los escolares mediante actividad física y se conocerá la eficacia de dichos recursos.

corporal (Antropometría, BIA y DEXA). Tesis doctoral. Universidad de Salamanca.

Alburquerque Sendín, F., Martín-Vallejo, F. J., García Talavera, P., Martín Gómez, M. E. \& Santos del Rey, M. (2010). Comparison of fat mass and fat-free mass between Anthropometry, BIA and DEXA in young 
females: are methods really interchangeable? European Journal of Anatomy, 14(3), 133-141.

Alvero, J. R., Diego, A. M., Fernández, V.J. \& García, J. (2004). Métodos de evaluación de la composición corporal: Tendencias actuales I. Archivos de Medicina del Deporte, 21(104), 535-538.

Alvero, J. R., Diego, A. M., Fernández, V.J. \& García, J. (2005a). Métodos de evaluación de la composición corporal: Tendencias actuales II. Archivos de Medicina del Deporte, 22(105), 45-49.

Alvero, J. R., Diego, A. M., Fernández, V.J. \& García, J. (2005b). Métodos de evaluación de la composición corporal: Tendencias actuales III. Archivos de Medicina del Deporte, 22(106), 121-127.

Alvero, J. R., Cabañas, M. D., Herrero, A., Martínez, L., Moreno, C., Porta, J., Sillero, M . \& Sirvent, J. E. (2010). Protocolo de valoración de la composición corporal para el reconocimiento médicodeportivo. Documento de consenso del Grupo Español de Cineantropometría (GREC) de la Federación Española de Medicina del Deporte (FEMEDE). Versión 2010. Archivos de Medicina del Deporte, 27(139), 330-344.

Bassey, E. J., Bryant, J. C., Clark, E., Fentem, P. H., Jones, P. R. M., Macdonald, I. A. \& Patrick, J. M. (1979). Factors affecting cardiac frequency during self paced walking - body-composition, age, sex and habitual activity. Journal of physiology, 291, 46-46.

Berral, F. J., Escribano, A., Berral, C. J., Delgado, C., Lancho, J. L. y De Rose, E. $\left(\begin{array}{cccc}1 & 9 & 9 & 1\end{array}\right)$. Comparative corporal composition study on the Faulkner an Kerr methods in athletes. 13th International Congress on Biomechanics. Perth. Australia.

Björntorp, P. (1992). Male fat distribution and cardiovascular risk. Blood Pressure, 1(suppl. 4), 17-19.

Donnelly, J. E., Jakicic, J. \& Gunderson, S. (1991). Diet and body-composition - effect of very low calorie diets and exercise. Sports medicine, 12(4), 237-249.

Espín Ríos, M. I., Pérez Flores, D., Sánchez Ruíz, J.F. \& Salmerón Martínez, D. (2013). Prevalencia de obesidad infantil en la Región de Murcia, valorando distintas referencias para el índice de masa corporal. Anales de Pediatría, 78, 6, 374-381.
Instituto Nacional de Estadística (2012). Encuesta Nacional de Salud 2011-2012: Índice de masa corporal en población infantil según sexo y grupo de edad. Población de 2 a 17 años.

Laskey, M. A. (1996). Dual-energy X-ray absorptiometry and body composition. Nutrition, $12(1), 45-51$.

Lebrun, C. E. I., van der Schouw, Y. T., de Jong, F. H., Pols, H. A. P., Grobbee, D. E. \& Larnberts, S. W. J. (2006). Relations between body composition, functional and hormonal parameters and quality of life in healthy postmenopausal women. Maturitas, 55(1), 82-92.

Logan, S. W., Scrabis-Fletcher, K., Modlesky, C. \& Getchell, N. (2011). The Relationship Between Motor Skill Proficiency and Body Mass Index in Preschool Children, Research Quarterly for Exercise and Sport, 82, 3, 442-448.

Moreno Villares, J. M. (2000). Técnicas de valoración de la composición corporal. Actas del XXIX Congreso Nacional Ordinario de Pediatría de la Asociación Española de Pediatría.

Organización Mundial de la Salud (2013). Simplified field tables: BMI-for-age girls and boys 2 to 5 years (percentiles).

Oria, E., Lafita, J., Petrina, E. \& Argüelles, I. (2002). Composición corporal y obesidad. Body composition and obesity. Anales del Sistema Sanitario de Navarra, 25(1), 91-102.

Ortega, E., Ortiz, I. M., Artés, E. M. (2009). Manual de Estadística Aplicada a las Ciencias de la Actividad Física y el Deporte. Murcia: Diego Marín.

Plonka, M., Toton-Morys, A., Adamski, P., Suder, A., Bielanski, W., Dobrzanska, M. J., Kaminska, A., Piorecka, B. \& Glodzik, J. (2011). Association of the physical activity with leptin blood serum level, body mass indices and obesity in schoolgirls. Journal of physiology and pharmacology, 62(6), 647-656.

Roche, A. F. (1992). Growth, maturation, and body composition. Cambridge: University Press.

Rosenfalck, A. M., Almdal, T., Gotfredsen, A. \& Hilsted, J. (1996). Body composition in normal subjects: Relation to lipid and glucose variables. International journal of obesity, 20(11), 1006-1013. 